\title{
Ecklonia cava Extract Containing Dieckol Suppresses RANKL-Induced Osteoclastogenesis via MAP Kinase/NF- $\kappa B$ Pathway Inhibition and Heme Oxygenase-1 Induction
}

\author{
Seonyoung $\mathrm{Kim}^{1}$, Seok-Seong Kang ${ }^{2}$, Soo-Im $\mathrm{Choi}^{3}$, Gun-Hee $\mathrm{Kim}^{3}$, and Jee-Young $\mathrm{Imm}^{1 *}$ \\ ${ }^{1}$ Department of Foods and Nutrition, Kookmin University, Seoul 02707, Republic of Korea \\ ${ }^{2}$ Department of Food Science and Biotechnology, Dongguk University, Ilsan 10326, Republic of Korea \\ ${ }^{3}$ Plant Resources Research Institute, Duksung Women's University, Seoul 01369, Republic of Korea
}

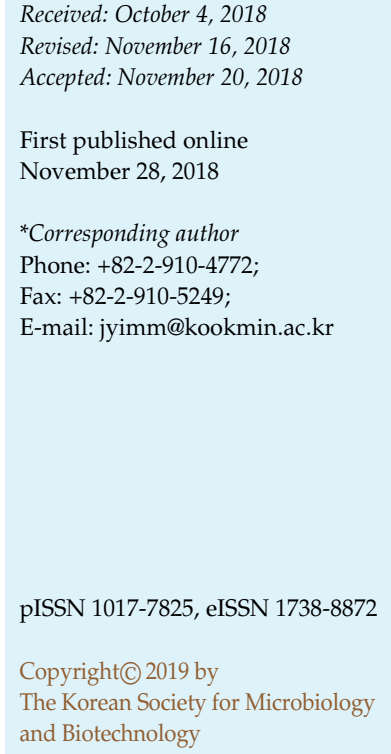

Ecklonia cava, an edible marine brown alga (Laminariaceae), is a rich source of bioactive compounds such as fucoidan and phlorotannins. Ecklonia cava extract (ECE) was prepared using $70 \%$ ethanol extraction and ECE contained $67 \%$ and $10.6 \%$ of total phlorotannins and dieckol, respectively. ECE treatment significantly inhibited receptor activator of nuclear factor- $\mathrm{kB}$ ligand (RANKL)-induced osteoclast differentiation of RAW 264.7 cells and pit formation in bone resorption assay $(p<0.05)$. Moreover, it suppressed RANKL-induced NF- $\mathrm{B}$ and mitogen-activated protein kinase signaling in a dose dependent manner. Downregulated osteoclast-specific gene (tartrate-resistant acid phosphatase, cathepsin $\mathrm{K}$, and matrix metalloproteinase-9) expression and osteoclast proliferative transcriptional factors (nuclear factor of activated T cells-1 and c-fos) confirmed ECE-mediated suppression of osteoclastogenesis. ECE treatment $(100 \mu \mathrm{g} / \mathrm{ml})$ increased heme oxygenase- 1 expression by 2.5 -fold and decreased intercellular reactive oxygen species production during osteoclastogenesis. The effective inhibition of RANKL-stimulated osteoclast differentiation and oxidative stress by ECE suggest that ECE has therapeutic potential in alleviating osteoclast-associated disorders.

Keywords: Ecklonia cava extract, osteoclast, bone resorption, MAP kinases, heme oxygenase-1

\section{Introduction}

The balance between bone-forming osteoblasts and boneresorbing osteoclasts is tightly regulated to maintain bone homeostasis and increased osteoclast differentiation is closely associated with the onset of bone related disease such as osteoporosis and periodontitis [1]. Osteoclasts are derived from the monocyte/macrophage lineage and differentiation from their precursors is initiated by receptor activator of nuclear factor- $\kappa B$ ligand (RANKL) expressed by osteoblasts [2]. The binding of RANKL to receptor activator of nuclear factor- $\mathrm{B}$ (RANK), located on the surface of osteoclast precursor cells, leads to activation of tumor necrosis factor-alpha (TNF) receptor-associated factor 6 and other downstream signaling molecules including nuclear factor- $\kappa \mathrm{B}(\mathrm{NF}-\kappa \mathrm{B})$, mitogen-activated protein kinase
(MAPK), and activator protein-1 (AP-1) [3]. The activation of these signaling molecules induces osteoclastic gene expressions such as tartrate-resistant acid phosphatase (TRAP), cathepsin K, and matrix metalloproteinase 9 (MMP-9) during osteoclastogenesis [4]. Thus, the suppression of osteoclast differentiation is an important target in the modulation of osteoclast-associated disorders.

Ecklonia cava, which is an edible marine brown alga (Laminariaceae), is a rich source of bioactive compounds such as fucoidan and polyphenols. It is widely distributed along the southern coast of Korea [5]. Phlorotannins are major phenolic compounds found in E. cava and have diverse oligomeric structures containing the phloroglucinol unit. These marine polyphenols have unique chemical structures and differ from terrestrial plant polyphenols, which are based on condensed hydrolysable tannins [6]. 
Eckol, 6,6'-bieckol, dieckol, and phlorofucofuroeckol A are major phlorotannin components [7]. Among these components, dieckol is one of the most potent bioactive compounds and effectively improves type II diabetes in $d b / d b$ mouse model via AMPK and Akt signaling pathways [8]. The open- chain trimeric phlorotannin, eckol, reduces $\mathrm{H}_{2} \mathrm{O}_{2}$-induced oxidative stress by increasing catalase expression in Chinese hamster lung fibroblast cells (V79-4) [9]. The hexameric phlorotannin, 6, 6'-bieckol strongly inhibits production of pro-inflammatory mediators such as iNOS and COX-2 and shows anti-inflammatory effect [10]. In addition, E. cava ethanol extract (ECE) has neuroprotective effects in BV2 microglia cells [11]. This strong antioxidative and ant-inflammatory activity of ECE suggests that it exerts suppressive effect on osteoclast formation and bone resorption. The present study was conducted to investigate the effect of ECE on RANKL-induced differentiation of osteoclasts and the molecular mechanisms underlying ECE-induced suppression of osteoclastogenesis.

\section{Materials and Methods}

\section{Materials}

E. cava extract (ECE) was kindly provided from Seojin Biotech Co. Ltd. (Korea) and dieckol (purity 99.5\%) was obtained from BotaMedi (Korea). Dulbecco's Modified Eagle's Medium (DMEM), alpha-minimum essential Eagle's medium ( $\alpha$-MEM), penicillinstreptomycin solution and fetal bovine serum (FBS) were purchased from Welgene Inc. (Korea). The high-capacity RNA-tocDNA kit, Taqman Universal Master Mix and Taqman gene expression assays were obtained from Applied Biosystems (USA). p38, ERK, NF-kB, $\beta$-actin and TBP antibodies were obtained from Cell Signaling Technology (USA) and JNK antibodies were obtained from Santa Cruz Biotechnology Inc. (USA). RANKL was purchased from ProSpec (Israel). Other reagents were of analytical grade and were purchased from Sigma-Aldrich Inc. (USA).

\section{Preparation of ECE}

E. cava was collected from July to September, 2017 in Jeju Island, Korea. The collected E. cava was thoroughly washed with purified water and air dried prior to extraction. Dried E. cava powder (50 100 mesh) was extracted with $70 \%(\mathrm{v} / \mathrm{v})$ ethanol for $12 \mathrm{~h}$ at $60^{\circ} \mathrm{C}$ under reflux condition. The clear supernatant was recovered from crude extract by continuous-centrifuge (J-1050A, Hanil Scimed Co., Ltd., Korea) at 12,000 × . Finally, ECE was obtained by lyophilization after solvent removal.

\section{Total Phlorotannin and Dieckol Content in ECE}

A modified Folin-Ciocalteu method [12] was used to analyze total phlorotannin content in ECE using phloroglucinol as the standard. Dieckol content in ECE was quantified using a Waters
HPLC system (Waters, USA) equipped with a CAPCELL PAK C18 column (Shiseido Com., Ltd., Japan, Shiseido $250 \times 4.6 \mathrm{~mm}, 5 \mu \mathrm{m})$. ECE was eluted by a gradient of solvents A $(0.1 \%, v / v$, TFA in water) and $\mathrm{B}(0.1 \%, \mathrm{TFA}, \mathrm{v} / \mathrm{v}$, in acetonitrile). The elution gradient was as follows: $0-10 \mathrm{~min}, 0-10 \% \mathrm{~B} ; 10-40 \mathrm{~min}, \mathrm{~B} 10-40 \%$; and $40-55 \mathrm{~min}, 40-10 \% \mathrm{~B}$. The flow rate was $1.0 \mathrm{ml} / \mathrm{min}$ and dieckol was detected at $230 \mathrm{~nm}$. Dieckol content in ECE was calculated using authentic standard curve.

\section{Cell Culture}

Murine macrophage RAW 264.7 cells were purchased from American Type Culture Collection (ATCC, USA) and were cultured in DMEM supplemented with $10 \%$ fetal bovine serum (FBS) and penicillin-streptomycin $(100$ units $/ \mathrm{ml})$ at $37^{\circ} \mathrm{C}$ in a humidified atmosphere under $5 \% \mathrm{CO}_{2}$. To differentiate RAW 264.7 cells into osteoclasts, the medium was replaced with $\alpha$-MEM containing RANKL $(50 \mathrm{ng} / \mathrm{ml})$ and M-CSF $(25 \mathrm{ng} / \mathrm{ml})$. The medium was changed every 2 days during a $4 \sim 10$ day incubation period. Cell viability was determined using the 3-(4,5-dimethylthiazol-2yl)-2,5-diphenyltetrzolium bromide (MTT) assay after 10 days in the presence of samples as previously described [13].

\section{Tartrate-Resistant Acid Phosphatase-Positive (TRAP (+)) Staining and TRAP Activity}

RAW 264.7 cells were seeded $\left(1 \times 10^{4}\right.$ cells/well) for $24 \mathrm{~h}$, and incubated for an additional 10 days in the medium containing RANKL (50 ng/ml), M-CSF ( $25 \mathrm{ng} / \mathrm{ml})$, and ECE. After osteoclast differentiation, the cells were fixed with $10 \%$ formalin for $5 \mathrm{~min}$, and TRAP (+) staining was performed using a staining kit (Cosmo Bio, Japan). Stained multi-nucleated osteoclast images were captured using an i304 e-scope (Macrotech, Korea). For the determination of TRAP activity, the cells were incubated for 4 days and lysed using Triton X-100 (0.05\%)/saline solution. After the cells were treated with $50 \mathrm{mM}$ citrate buffer ( $\mathrm{pH}$ 4.7) containing $10 \mathrm{mM}$ sodium tartrate and $10 \mathrm{mM}$-nitrophenylphosphate for $30 \mathrm{~min}$ at $37^{\circ} \mathrm{C}$, absorbance was measured at $405 \mathrm{~nm}$ using a microplate reader (Biotek Instruments Inc., USA). TRAP activity was expressed as a percentage of the control (only RANKLtreated)

\section{Bone Resorption Assay}

The effect of ECE on osteoclast-mediated bone resorption was determined using a fluoresceinated calcium phosphate-coated plate (Cosmo Bio). RAW 264.7 cells grown in phenol red-free-DMEM were seeded on the plate $\left(1 \times 10^{4}\right.$ cells/well) and incubated for 6 days in the presence of $50 \mathrm{ng} / \mathrm{ml}$ RANKL, M-CSF ( $25 \mathrm{ng} / \mathrm{ml})$ and ECE. After 6 days, the medium $(100 \mu \mathrm{l})$ was taken and mixed with the resorption assay buffer (Cosmo Bio). Fluorescence intensity was measured at an excitation wavelength of $485 \mathrm{~nm}$ and emission wavelength of $535 \mathrm{~nm}$ using a microplate reader (Biotek Instruments). Representative images from each treatment were captured by light microscopy after washing with sodium hypochlorite $(5 \%, \mathrm{w} / \mathrm{v})$. 


\section{Reactive Oxygen Species (ROS) Production}

The effect of ECE on intracellular ROS production was determined using 2',7'-dichlorodihydrofluorescein-diacetate $\left(\mathrm{H}_{2} \mathrm{DCF}-\mathrm{DA}\right)$. Cells were treated with the indicated concentration of samples for $2 \mathrm{~h}$, and they were then stimulated with RANKL $(100 \mathrm{ng} / \mathrm{ml})$ and M-CSF ( $50 \mathrm{ng} / \mathrm{ml})$ for $1 \mathrm{~h}$. After incubation with $\mathrm{H}_{2}$ DCF-DA $(50 \mu \mathrm{M})$ for $30 \mathrm{~min}$ at $37^{\circ} \mathrm{C}$, the cells were washed and resuspended in Hanks' balanced salt solution. Fluorescence intensity was measured using a microplate reader (Biotek Instruments) at $485 \mathrm{~nm}$ excitation and $528 \mathrm{~nm}$ emission.

\section{RNA Extraction and Quantitative Real-Time PCR (qRT-PCR)}

Total RNA was extracted and qRT-PCR was performed using a StepOne Plus real-time RCR system (Applied Biosystems) as previously described [13]. The following primers were used in the analysis; $\beta$-actin (Mm00607939_s1), TRAP (Mm00475698_m1), cathepsin K (Mm00484039_m1), MMP-9 (Mm00442991_m1), NFATc1 (Mm00479445_m1), and c-fos (Mm00487425_m1). Taqman probes (dual-labeled with 6-carboxyfluorescein as the $5^{\prime}$-reporter and 3' TAMRA quencher) were used for assays. The relative quantity of target mRNA (TRAP, cathepsin K, MMP-9, NFATc1 and c-fos) was determined using the comparative $\mathrm{C}_{\mathrm{T}}$ method by normalizing to the value of the housekeeping gene $\beta$-actin. All reactions were run in triplicate.

\section{Western Blotting Analyses}

Cytoplasmic and nuclear protein extraction were done using commercial extraction reagents (Thermo Scientific, USA) according to the manufacturer's protocol. Equal amounts of proteins in each sample were separated on a 10\% SDS-PAGE gels and then transferred onto a polyvinylidene fluoride membranes (Millipore, USA). After blocking with 5\% BSA in tris-buffered saline for $1 \mathrm{~h}$ at room temperature, the membranes were incubated with appropriate primary antibodies (dilution ratio 1:1000) overnight at $4^{\circ} \mathrm{C}$. Targeted protein bands were visualized with horse radish peroxidase-conjugated secondary antibodies by an enhanced chemiluminescence detection system (Bio-Rad, USA).

\section{Statistical Analysis}

Experiments were conducted in triplicate, and data were expressed as mean \pm standard deviations (SD). Statistical analyses were performed using SPSS (SPSS, Inc., USA). When data showed significant differences $(p<0.05)$ in one-way analysis of variance, Duncan's multiple comparisons test was used to find significant differences among treatment means.

\section{Results and Discussion}

\section{Phlorotannin and Dieckol Content of ECE}

The yield of ECE after ethanol (70\%, v/v) extraction was $2.8 \pm 0.2 \%$ (dry weight basis) and total phlorotannin content of ECE was $67 \pm 1 \%$. The dieckol content in ECE was $10.6 \pm$
$0.1 \%$ (data not shown). The yield and composition of extracted phytochemicals varied depending on the source, extraction conditions, and procedures. Total pholorotannin content of ECE prepared with 95\% ethanol was about 58\% [12]. Shin et al. [14] reported that total phlotannin content in the extracts obtained either by aqueous or 30\% ethanol extraction were 21 and 45\%, respectively. Lee et al. [15] isolated phlorotannin compounds in ECE using centrifugal partition chromatography. The dieckol content of ECE was about $8 \%$ and was comparable to that obtained in the present study (data not shown).

\section{Effect of ECE on Osteoclast Differentiation and Bone Resorption in RANKL-Stimulated RAW 264.7 Cells}

The effect of ECE on RANKL-induced osteoclast differentiation was examined using TRAP staining and TRAP activity assay. Osteoclasts are formed from their precursor cells such as monocytes and macrophages, and express TRAP [2]. Thus, TRAP staining was used to identify multinucleated osteoclasts and TRAP activity assay was performed as a solid cytochemical marker for osteoclasts.

The formation of mature multinucleated osteoclasts from RAW 264.7 cells was observed upon RANKL treatment while the extent of osteoclast formation was significantly decreased with ECE treatment (Fig. 1A). TRAP activity in serum significantly increased during osteoporosis; this is associated with increased bone resorption [16]. As shown in Fig. 1B, TRAP activity of multinucleated TRAP (+) cells also significantly decreased by ECE treatment while cytotoxic effect of ECE was not observed in the tested concentrations (Fig. 1C).

Rahim et al. [17] reported that phloroglucinol, which is a monomeric unit of dieckol, was able to inhibit osteoclastogenesis by suppressing RANKL-RANK interaction based on the changes of bonding energy of inter-protein docking analysis. RANKL/RANK/osteoprotegerin (OPG) signaling plays a critical role in bone remodeling and suppression and this is effective in various bone diseases with increased bone loss in animal models [18]. Recently, diphlorethohydroxycarmalol, a phlorotanin isolated from brown alga (Ishige okamurae) brought about an antiosteoclastogenetic effect by suppressing RANKL/NF- $\kappa B$ signaling [19].

Mature osteoclasts have typical bone-resorbing activity. The effect of ECE treatment on bone resorption activity was analyzed using a calcium phosphate-coated culture plate. Relative fluorescence intensity due to the formation of resorption pits also significantly decreased with ECE treatment (Fig. 2). This result suggests that ECE effectively 
A
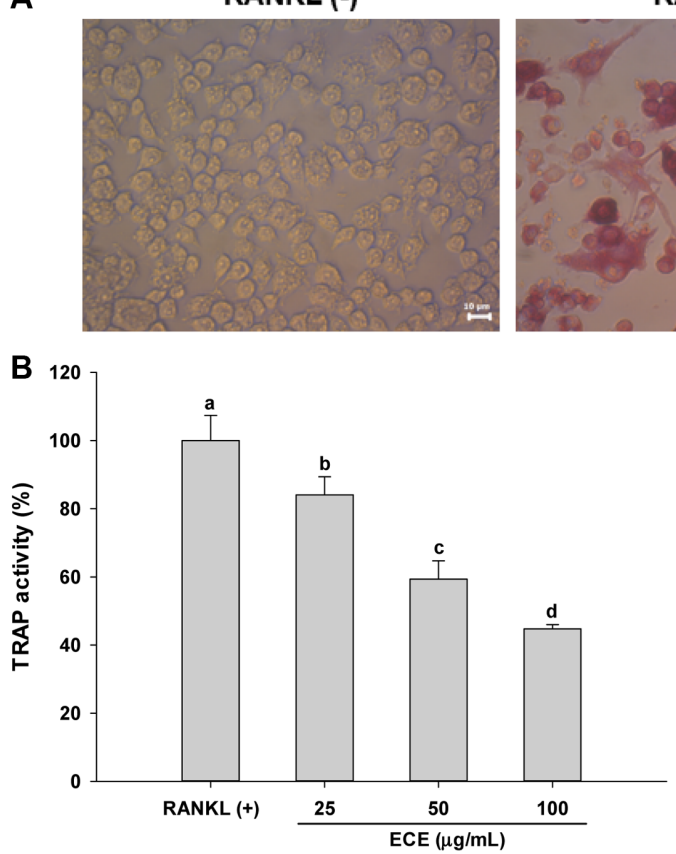

RANKL (+)
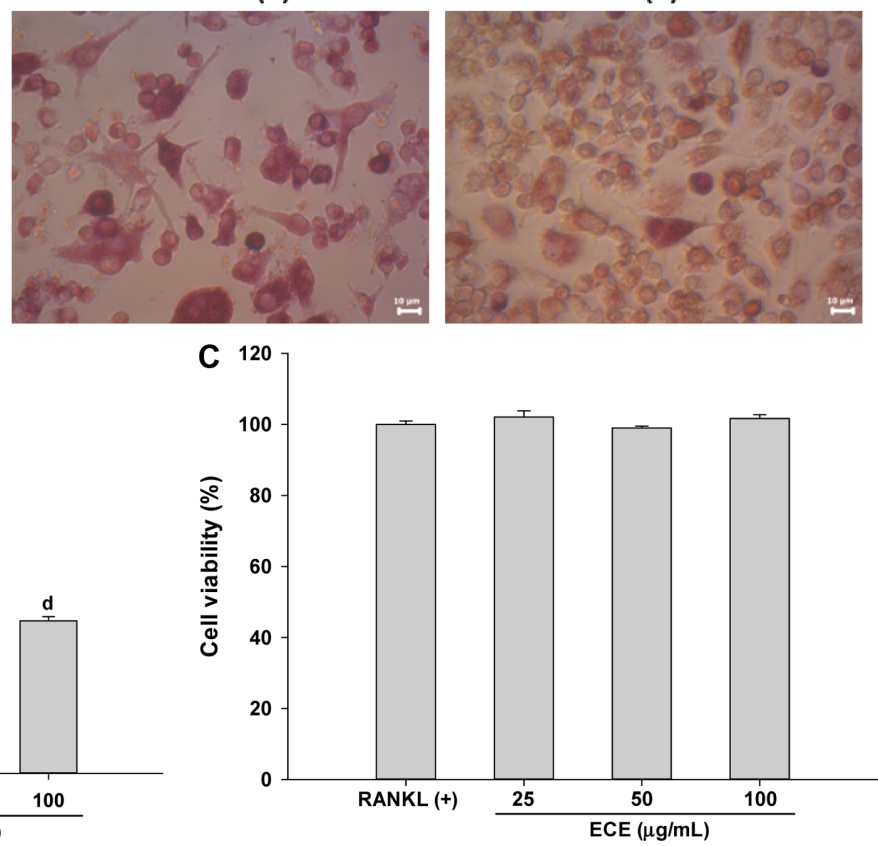

Fig. 1. Effects of ECE on TRAP formation and activity in RANKL-induced RAW 264.7 macrophages.

ECE: Ecklonia cava extract, RANKL: receptor activator of nuclear factor kappa-B ligand. (A) TRAP staining image. Cells were incubated for 10 days in the presence of RANKL (50 ng/ml), M-CSF $(25 \mathrm{ng} / \mathrm{ml})$, and sample (ECE $100 \mu \mathrm{g} / \mathrm{ml})$. TRAP staining was observed using a microscope and TRAP (+) stained multinucleated cells containing $\geq 3$ nuclei were considered osteoclasts. Scale bar unit: $10 \mu \mathrm{m}$. (B) TRAP activity. Activity was determined after 4 days of RANKL treatment. (C) Cytotoxicity. Cell viability was determined at 10 days using the MTT assay. Bars with different letters indicate significant differences at $p<0.05$.

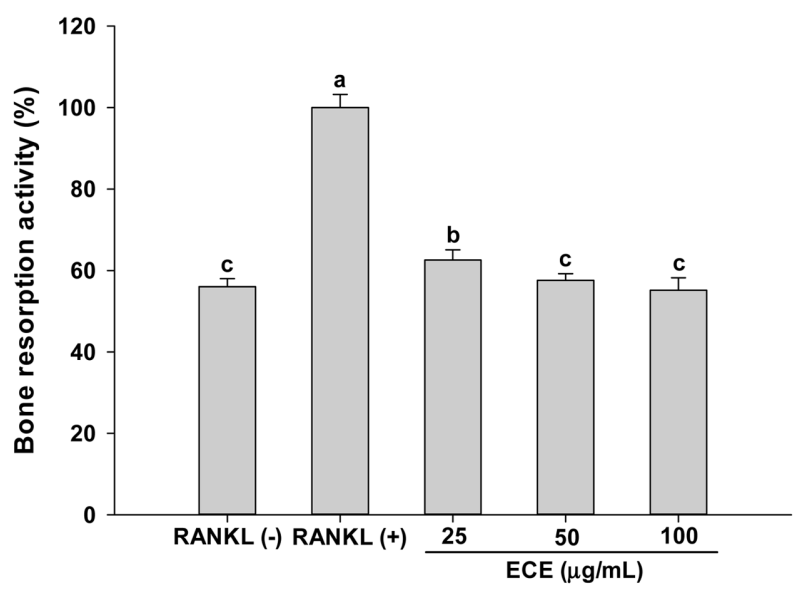

Fig. 2. Effect of ECE on bone resorption of RANKL-induced RAW 264.7 macrophages.

Cells were incubated for 6 days on fluoresceinamine-labeled chondroitin sulfate-labeled calcium phosphate-coated plates in the presence of RANKL $(50 \mathrm{ng} / \mathrm{ml}), \mathrm{M}-\mathrm{CSF}(25 \mathrm{ng} / \mathrm{ml})$, and samples. Bone absorption activity was evaluated by measuring the fluorescence intensity produced by the decomposition of calcium phosphate with fluorescence. Bars with different letters indicate significant differences at $p<0.05$. inhibited RANKL-induced bone resorptive pit formation. Based on these results, ECE treatment actively suppresses osteoclast differentiation and bone resorption.

\section{Effect of ECE on Expression of Osteoclast Specific Genes and Transcriptional Factors}

The effect of ECE on osteoclast specific genes such as TRAP, cathepsin K, and MMP-9 were examined using qRTPCR. The mRNA expression levels of all analyzed osteoclastspecific genes increased with RANKL stimulation whereas they were significantly downregulated with ECE treatment in a dose dependent manner $(p<0.05$, Fig. 3A). These results indicate that the suppression of osteoclastogenesis by ECE treatment are regulated by transcriptional level. MMP-9 and cathepsin K are highly expressed proteolytic enzymes in human osteoclasts and play an important role in bone resorption [4]. Thus, decreased gene expression of cathepsin K and MMP-9 reflects decreased bone resorption, and this was confirmed by the result of the bone resorption assay (Fig. 2). Kim et al. [12] reported that ECE showed strong human inhibitory activities of MMPs including MMP-2 and MMP-9 and that the potency of ECE was 
A
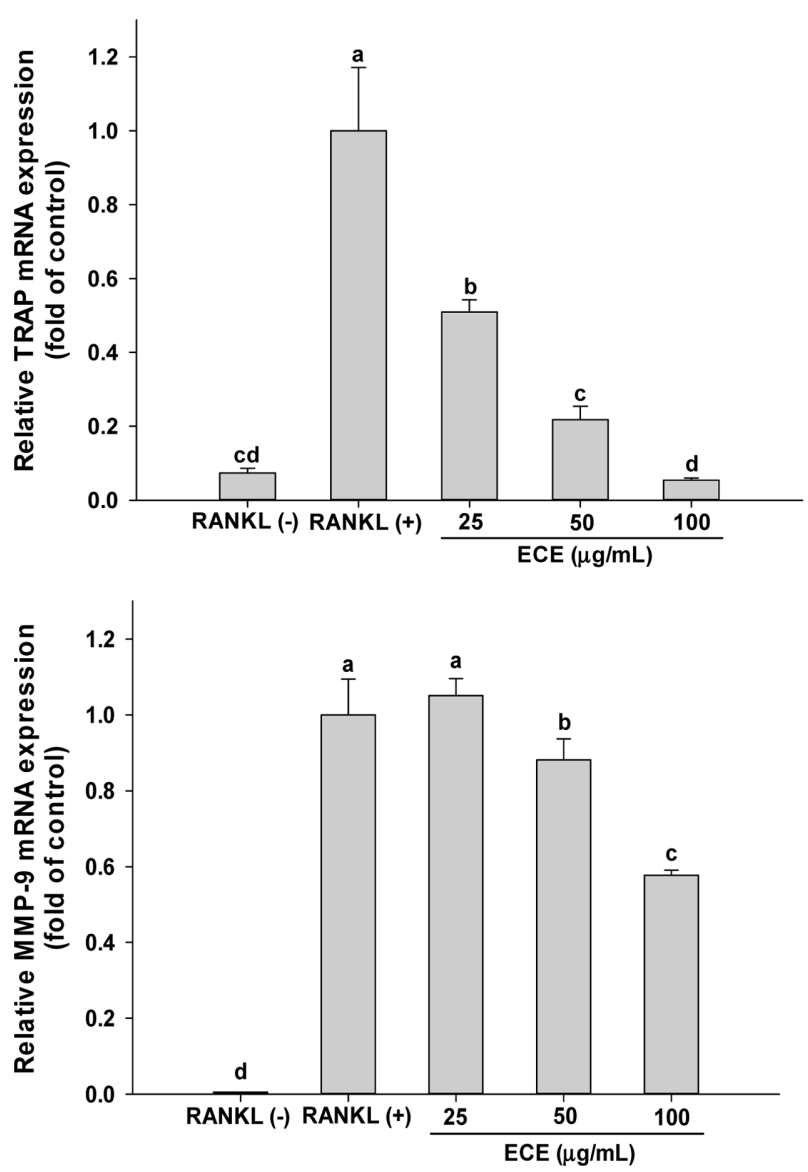
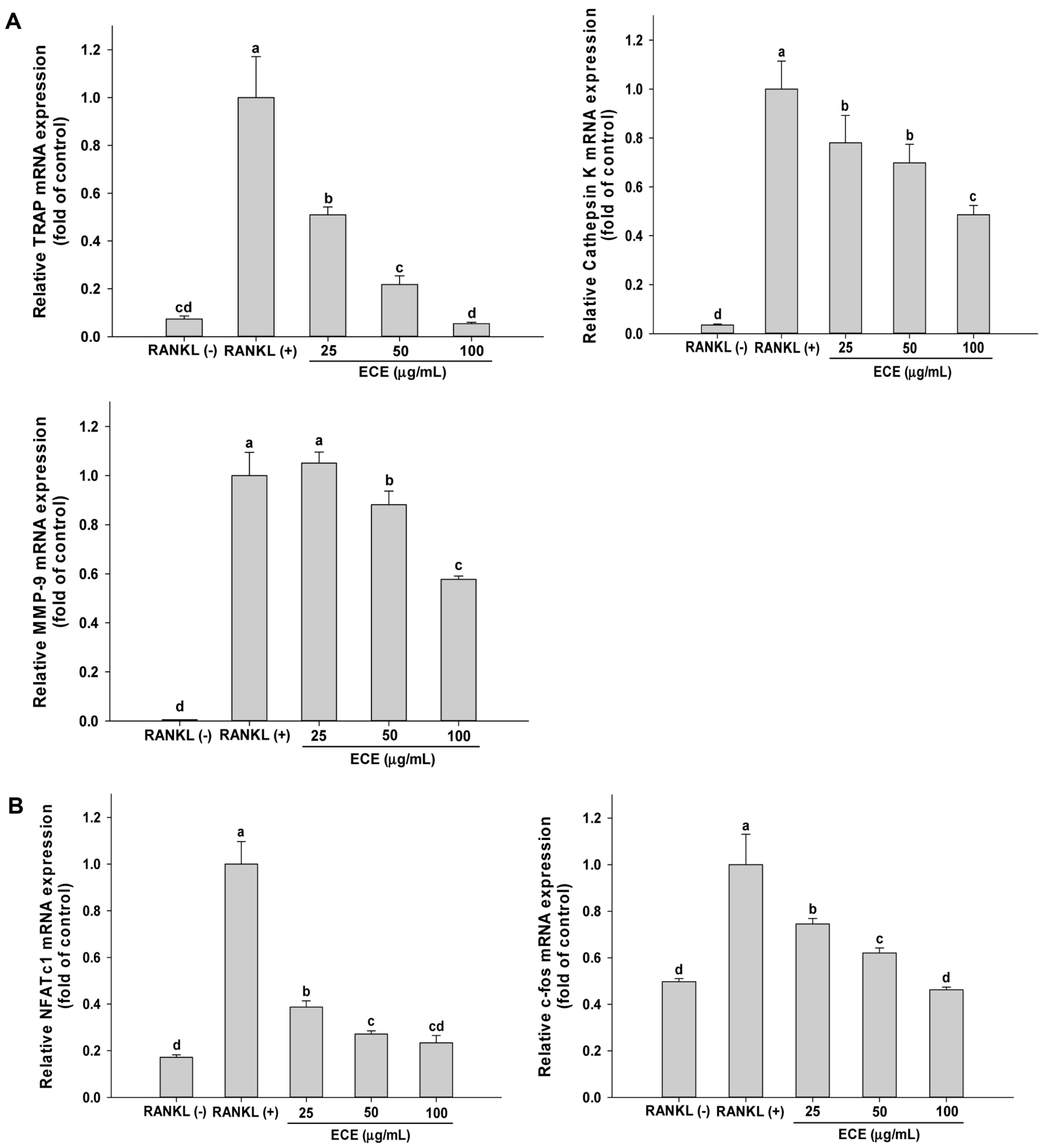

Fig. 3. Effect of ECE on osteoclast-specific genes and transcriptional factor expression levels in RANKL-induced RAW 264.7 macrophages.

TRAP: tartrate-resistant acid phosphatase, MMP-9: matrix metallopeptidase-9. Cells were incubated for 2 days in the presence of RANKL (50 ng/ml), M-CSF ( $25 \mathrm{ng} / \mathrm{ml}$ ), and samples. (A) Osteoclast-specific genes (TRAP, cathepsin K and MMP-9) expression levels and (B) Osteoclast proliferationrelated transcriptional factor (NFATc- 1 and c-fos) expression levels were analyzed using qPCR and normalized to that of $\beta$-actin. Bars with different letters indicate significant differences at $p<0.05$.

comparable to that of doxycycline which is used as a therapeutic agent.
Cell differentiation requires induction of target genes necessary for survival and maturation and transcription 
factors coordinate in this physiological process [20]. To gain insight regarding the transcriptional program induced by RANKL, mRNA expression of two key transcription factors, NFATc1 and c-fos were examined. As presented in Fig. 3B, mRNA expressions of NFATc1 and c-fos significantly decreased in response to ECE treatment. These results suggest that inhibition of osteoclast-specific transcriptional factors are involved in the ECE-mediated suppression of osteoclastogenesis.

NFATc1 is the master regulator of osteoclastogenesis since it controls expression of a series of osteoclast-specific genes related to adhesion/migration ( $\beta 3$ integrin and $C$ Src), acidification (ATP6i and CLC7), and degradation of bone matrix (cathepsin K and MMP-9) [21]. Takayanagi et al. [20] reported that the expression of NFATc1 in the cytoplasm was observed at $24 \mathrm{~h}$ after RANKL stimulation and its nuclear translocation was dominant at $48 \mathrm{~h}$. c-fos binds to the promoter region of NFATc1 and this complexation is required for induction and activation of NFATc1 in RANKL-induced osteoclastogenesis [22].

\section{Effect of ECE on RANKL-Induced Osteoclast Signaling Pathway}

Mitogen-activated protein kinases (MAPK) including p38MAPK, c-Jun N-terminal kinase (JNK), and extracellular signal-regulated kinases (ERK) modulate several biological processes such as inflammatory responses, and are regulated by RANKL [23]. This observation suggests that regulation of MAPK can be a potential target for RANKLmediated osteoclastogenesis. To examine the effects of ECE on RANKL-induced osteoclast signaling, changes in phosphorylation of MAPK were analyzed using western blotting. As shown in Fig. 4A, ECE treatment significantly inhibited phosphorylation of ERK1/2, p38, and JNK $(p<0.05)$. Ikeda et al. [24] examined the role of c-Jun signaling in the regulation of the NFAT family and RANKLinduced osteoclast differentiation using transgenic mice. They reported that a partnership between c-Jun/c-fos and the NFAT family is essential for differentiating osteoclasts and that they mutually promoted osteoclastogenesis.

$\mathrm{NF}-\kappa \mathrm{B}$ is a transcriptional factor that controls the early stage of RANKL-induced osteoclast differentiation leading to activation of c-fos and NFATc1. This event subsequently initiates the inflammation process [25]. The effect of ECE treatment on nuclear NF- $\mathrm{kB}$ expression in the nucleus was analyzed. As shown in Fig. 4B, the expression of NF- $\mathrm{BB}$ significantly decreased in a dose dependent manner. This result indicates that ECE counteracts RANKL-mediated osteoclastogenesis by blocking activation. Taken together, ECE actively inhibited osteoclastogenesis by downregulation of RANKL-mediated MAPK and NF- $\mathrm{KB}$ signaling.

Kim and Kim [26] reported that LPS-stimulated NF- $\kappa B$ activation and pro-inflammatory cytokine (TNF- $\alpha$, IL-1b, IL-6, and $\mathrm{PGE}_{2}$ ) production were significantly decreased in the presence of phloroglucinol in RAW 264.7 macrophages. However, only JNK signaling was significantly inhibited by phloroglucinol. In case of other phloroglucinol derivatives such as phlorofucofuroeckol A and dieckol, a strong suppressive effect was exerted on LPS-stimulated p38 MAPK activation in RAW 264.7 and BV2 microglial cells $[27,28]$. These previous observations suggest that NF-кB nuclear translocation can be modulated through multiple MAPK signaling pathways and different phlorotannin components may exert different effects. The inhibition of all 3 types of MAPK signaling by ECE might be related to diverse phlorotannin components in ECE.

\section{Effects of ECE on ROS Production and HO-1 Induction}

The effect of ECE treatment on RANKL-induced ROS production was measured using 2',7'-dichlorofluoresceindiacetate (DCF-DA). DCF-DA is converted to $2^{\prime}, 7^{\prime}$ dichlorodihydro-fluorescein $\left(\mathrm{H}_{2} \mathrm{DCF}\right)$ by cellular esterase and is rapidly oxidized to fluorescent $2^{\prime}, 7^{\prime}$-dichlorofluorescein (DCF). The accumulated $2^{\prime}, 7^{\prime}$-DCF in the cell reacts with ROS [29]. The intracellular ROS production of RAW264.7 cells substantially increased upon RANKL exposure, while the fluorescence intensities of samples significantly decreased in response to ECE treatment (Fig. 5A). Li et al. [7] compared antioxidant activities of seven phlorotannins isolated from E. cava using various antioxidant methods. All phlorotannins have antioxidant activities, and 6,6' bieckol and dieckol consistently showed stronger activity than other phlorotannin derivatives in different kinds of antioxidant assays.

ROS play an important role in bone remodeling and the onset of bone disease by promoting osteoclastogenesis [30]. Intercellular ROS are primarily produced in the form of superoxide anions by NADPH oxidase (Nox 1) located in the cell membrane and RANKL-induced activation of Nox 1 and mitochondrial dysfunction are responsible for increased ROS production [31, 32]. Kwon et al. [33] reported that Nox1 requires the effector protein Rac1 for its activation and the inhibition of Nox 1 complex formation with Rac 1 is closely related to anti-osteocalstic effect. Park and Jeon [34] reported that dieckol isolated from $E$. cava effectively suppresses invasion of HT 1080 cells by inhibiting Rac1- 
A
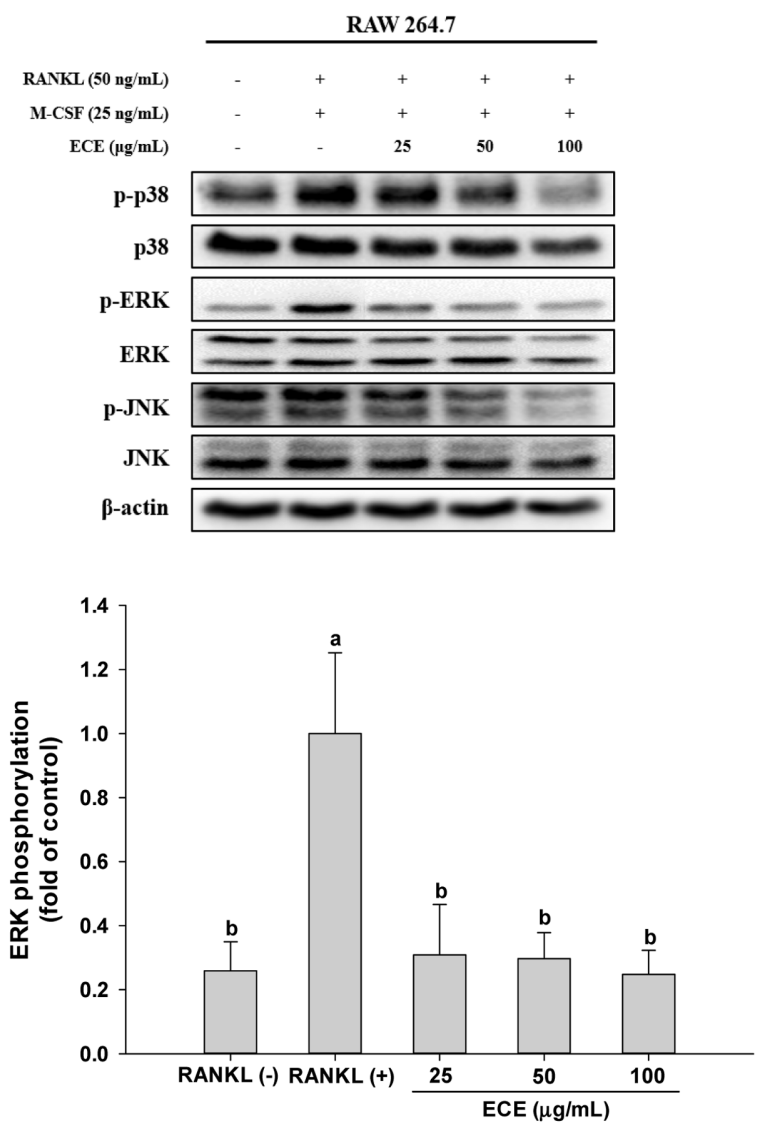
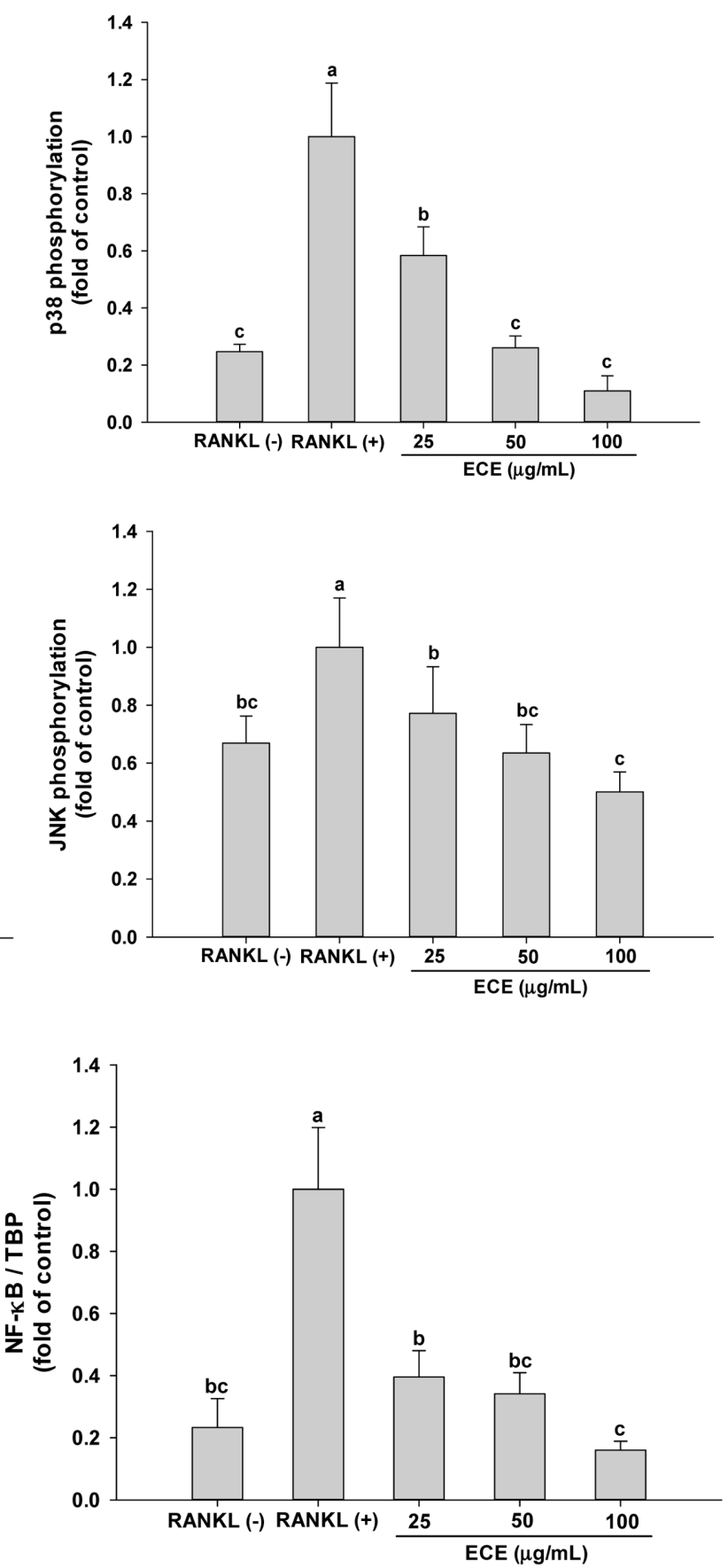

Fig. 4. Effects of ECE on protein expression associated with MAP kinases and nuclear NF-кB in RANKL-induced RAW 264.7 macrophages.

MAP kinases: mitogen-activated protein kinases (p38, ERK, and JNK). Cells were incubated for $24 \mathrm{~h}$ in the presence of RANKL (50 ng/ml), M-CSF $(25 \mathrm{ng} / \mathrm{ml})$, and samples. (A) The phosphorylation level of MAP kinases was analyzed using western blots and normalized to those of $\beta$-actin. (B) Nuclear protein lysates were prepared for the analysis of NF- $\kappa$ B and NF- $\kappa$ B expression was normalized to that of TBP. Bars with different letters indicate significant differences at $p<0.05$.

ROS signaling. Bai et al. [35] reported that increased intercellular ROS stimulated phosphorylation of MAPK
(ERK) and increased RANKL expression in mouse osteoblasts. These results suggest that inhibition of 


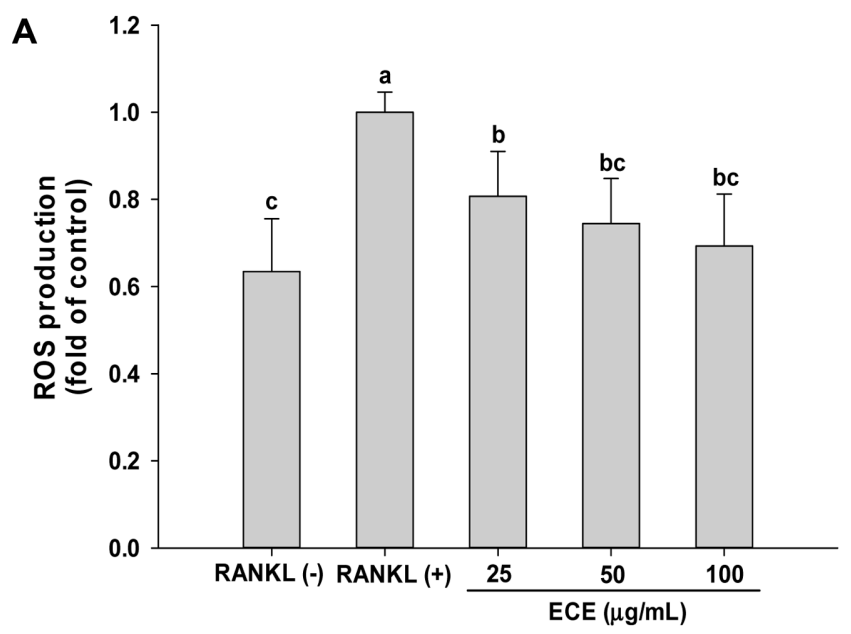

B
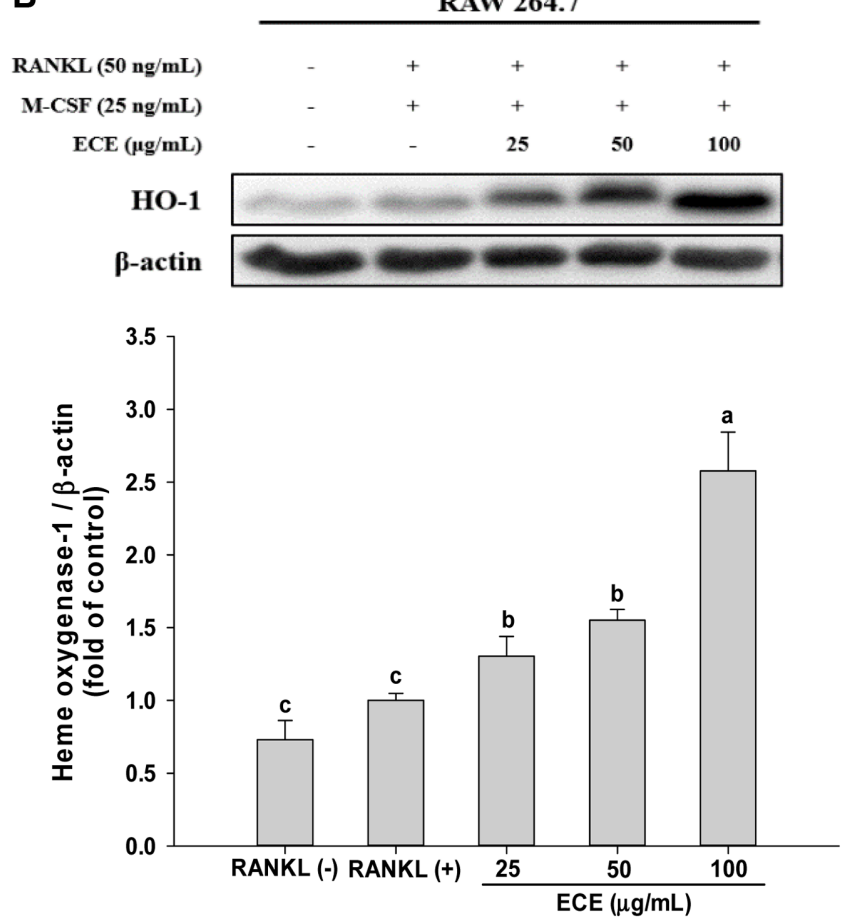

Fig. 5. Effects of ECE on ROS production and HO-1 expression in RANKL-induced RAW 264.7 macrophages.

HO-1: heme oxygenase-1. (A) Intercellular ROS production was determined using 2', $7^{\prime}$-dichlorodihydrofluorescein-diacetate $\left(\mathrm{H}_{2} \mathrm{DCF}-\right.$ DA). Cells were treated with indicated concentrations of samples for $2 \mathrm{~h}$ and then stimulated with RANKL $(100 \mathrm{ng} / \mathrm{ml})$ and M-CSF $(50 \mathrm{ng} / \mathrm{ml})$ for $1 \mathrm{~h}$. (B) HO-1 expression was analyzed using western blots and normalized to those of $\beta$-actin. Cells were incubated for $24 \mathrm{~h}$ in the presence of RANKL $(50 \mathrm{ng} / \mathrm{ml}), \mathrm{M}-\mathrm{CSF}(25 \mathrm{ng} / \mathrm{ml})$, and samples. Bars with different letters indicate significant differences at $p<0.05$.

osteoclastogenesis by ECE is partly due to suppression of ROS and phlorotannins such as dieckol contributing to decreased ROS-mediated bone resorption.

Heme oxygenase $(\mathrm{HO})$ is a transcriptionally upregulated antioxidant enzyme and only $\mathrm{HO}-1$ is inducible in response to cellular stress such as inflammation and oxidative stress. HO-1 plays a cytoprotective role in cellular stress conditions and the induction of HO-1 effectively inhibits RANKL-mediated osteoclastogenesis [36]. As presented in Fig. 5B, HO-1 expression increased by ECE treatment and was 2.5-fold greater than that of control at $100 \mathrm{mg} / \mathrm{ml}$. Consistent with the present study, eckol, a major phlorotannin component of E. cava, led to incresaed HO-1 expression in lung fibroblast (V79-4) cells [37]. The absence of $\mathrm{HO}-1$ resulted in decreased bone mass, and elevated serum ROS levels, and increased osteoclast numbers in HO-1 knockout mice and bone marrow cells [38]. The modulation of HO-1 was more effective than that of MAPK signaling to decrease the expression of inflammatory mediators ( $\mathrm{PGE}_{2}$, cyclooxygenase-2, and inducible nitric oxide) in nicotine and LPS-stimulated human periodontal ligament cells [39]. Based on these results, the authors suggested that HO-1 induction can be a valuable therapeutic target for alleviating periodontal diseases.

ECE containing dieckol effectively suppressed differentiation and bone resorption of osteoclasts via suppression of RANKL-induced NFkB and MAPK signaling. Downregulation of osteoclast-specific gene expression (TRAP, cathepsin K, and MMP-9) and osteoclast proliferative transcriptional factors (NFATc1 and c-fos) confirmed ECE-mediated suppression of osteoclastogenesis. ECE treatment also significantly increased HO-1 expression, which counteracts excessive ROS production. The inhibition of osteoclast differentiation and RANKL- stimulated oxidative stress by ECE suggest that ECE possesses therapeutic potential for the alleviation of osteoclast-associated disorders, such as periodontitis and osteoporosis. Experimental work on the efficacy validation of ECE on periodontitis using an animal model is in progress.

\section{Acknowledgment}

This research was part of a project titled "Development of Global Senior-friendly Health Functional Food Materials from Marine Resources (No. 20170297)" funded by the Ministry of Oceans and Fisheries, Korea.

\section{Conflict of Interest}

The authors have no financial conflicts of interest to declare. 


\section{References}

1. Asagiri M, Takayanagi H. 2007. The molecular understanding of osteoclast differentiation. Bone 40: 251-264.

2. Vaananen HK, Laitala-Leinonen T. 2008. Osteoclast lineage and function. Arch. Biochem. Biophys. 473: 132-138.

3. Wagner EF, Eferl R. 2005. Fos/AP-1 proteins in bone and the immune system. Immunol. Rev. 208: 126-140.

4. Logar DB, Komadina R, Prezelj J, Ostanek B, Trost Z, Marc J. 2007. Expression of bone resorption genes in osteoarthritis and in osteoporosis. J. Bone Miner Metab. 25: 219-225.

5. Wijesekara I, Yoon NY, Kim SK. 2010. Phlorotannins from Ecklonia cava (Phaeophyceae): biological activities and potential health benefits. Biofactors 36: 408-414.

6. Shibata T, Kawaguchi S, Hama Y, Inagaki M, Yamaguchi K, Nakamura T. 2004. Local and chemical distribution of phlorotannins in brown algae. J. Appl. Phycol. 16: 291-296.

7. Li Y, Qian ZJ, Ryu B, Lee SH, Kim MM, Kim SK. 2009. Chemical components and its antioxidant properties in vitro: an edible marine brown alga, Ecklonia cava. Bioorg. Med. Chem. 17: 1963-1973.

8. Kang MC, Wijesinghe WAJP, Lee SH, Kang SM, Ko SC, Yang $\mathrm{X}$, et al. 2014. Dieckol isolated from brown seaweed Ecklonia cava attenuates type II diabetes in $d b / d b$ mouse model. Food Chem. Toxicol. 158: 433-437.

9. Kang KA, Chae S, Lee KH, Zhang R, Jung MS, Kim SY, et al. 2005. Eckol isolated from Ecklonia cava attenuates oxidative stress induced cell damage in lung fibroblast cells. FEBS Lett. 579: 6295-6304.

10. Yang YI, Shin HS, Kim SH, Park WY, Lee KT, Choi JH. 2012. 6,6'-Bieckol, isolated from marine alga Ecklonia cava, suppressed LPS-induced nitric oxide and PGE2 production and inflammatory cytokine expression in macrophages: The inhibition of NFKB. Int. Immunopharmacol. 12: 510-517.

11. Jung WK, Heo SJ, Jeon YJ, Lee CM, Park YM, Byun HG, et al. 2009. Inhibitory effects and molecular mechanism of dieckol isolated from marine brown alga on COX-2 and iNOS in microglial cells. J. Agric. Food Chem. 57: 4439-4446.

12. Kim MM, Van Ta Q, Mendis E, Rajapakse N, Jung WK, Byun HG, et al. 2006. Phlorotannins in Ecklonia cava extract inhibit matrix metalloproteinase activity. Life Sci. 79: 14361443

13. Lee D, Imm JY. 2017. AMP kinase activation and inhibition of nuclear factor-kappa B (NF-kB) translocation contribute to the anti-inflammatory effect of tricin. J. Food Biochem. 41: e12293.

14. Shin HC, Hwang HJ, Kang KJ, Lee BH. 2006. An antioxidative and antiinflammatory agent for potential treatment of osteoarthritis from Ecklonia cava. Arch. Pharm. Res. 29: 165-171.

15. Lee JH, Ko JY, Oh JY, Kim CY, Lee HJ, Kim J, et al. 2014. Preparative isolation and purification of phlorotannins from Ecklonia cava using centrifugal partition chromatography by one-step. Food Chem. 158: 433-437.

16. Hayman AR. 2008. Tartrate-resistant acid phosphatase (TRAP) and osteoclast/immune cell dichotomy. Autoimmunity 41: 218-223.

17. Rahim AH, Setiawan B, Dewi FRP, Noor Z. 2015. Regulation by phloroglucinol of Nrf2/Maf-mediated expression of antioxidant enzymes and inhibition of osteoclastogenesis via the RANKL/RANK signaling pathway: In silico study. Acta Infom. Med. 23: 228-232.

18. Boyce BF, Xing L. 2008. Functions of RANKL/RANK/OPG in bone modeling and remodeling. Arch. Biochem. Biophys. 473: 139-146.

19. Ihn HJ, Kim JA, Cho HS, Shin HI, Kim GY, Choi YH, et al. 2017. Diphlorethohydroxycarmalol from Ishige okamurae suppresses osteoclast differentiation by downregulating the NF-кB signaling pathway. Int. J. Mol. Sci. 18: 12.

20. Takayanagi H, Kim S, Koga T, Nishina H, Isshiki M, Yoshida $\mathrm{H}$, et al. 2002. Induction and activation of the transcription factor NFATc1 (NFAT2) integrate RANKL signaling in terminal differentiation of osteoclasts. Dev. Cell 3: 899-901.

21. Zhao Q, Wang X, Liu Y, He A, Jia R. 2010. NFATc1: Functions in osteoclasts. Int. J. Biochem. Cell Biol. 42: 576-579.

22. Matsuo K, Galson DL, Zhao C, Peng L, Laplace C, Wang KZ, et al. 2004. Nuclear factor of activated T-cells (NFAT) rescues osteoclastogenesis in precursors lacking c-Fos. J. Biol. Chem. 272: $26475-26480$.

23. Wada T, Nakashima T, Hiroshi N, Penninger JM. 2006. RANKL RANK signaling in osteoclastogenesis and bone disease. Trends Mol. Med. 12: 17-25.

24. Ikeda F, Nishimura R, Matsubara T, Tanaka S, Inoue JI, Reddy SV, et al. 2004. Critical roles of c-Jun signaling in regulation of NFAT family and RANKL-regulated osteoclast differentiation. J. Clin. Invest. 114: 475-484.

25. Yamashita T, Yao Z, Li F, Zhang Q, Badell IR, Schwarz EM, et al. 2007. NF-кB p50 and p52 regulate receptor activator of NF-кB Ligand (RANKL) and tumor necrosis factor-induced osteoclast precursor differentiation by activating c-Fos and NFATc1. J. Biol. Chem. 282: 18245-18253.

26. Kim MM, Kim SK. 2010. Effect of phloroglucinol on oxidative stress and inflammation. Food Chem. Toxicol. 48: 2925-2933.

27. Kim AR, Lee MS, Shin TS, Hua H, Jang BC, Choi JS, et al. 2011. Phlorofucofuroeckol A inhibits the LPS-stimulated iNOS and COX-2 expressions in macrophages via inhibition of NF-kB, Akt, and p38 MAPK. Toxicol. In Vitro 25: 1789-1795.

28. Jung WK, Ahn YW, Lee SH, Choi YH, Kim SK, Yea SS, et al. 2009. Ecklonia cava ethanolic extracts inhibit lipopolysaccharideinduced cyclooxygenase- 2 and inducible nitric oxide synthase expression in BV2 microglia via the MAP kinase and NF- $\mathrm{KB}$ pathways. Food Chem. Toxicol. 47: 410-417.

29. Keller A, Mohamed A, Drose S, Brandt U, Fleming I, Brandes RP. 2004. Analysis of dichlorodihydrofluorescein and dihydrocalcein as probes for the detection of intracellular 
reactive oxygen species. Free Radic. Res. 38: 1257-1267.

30. Wauquier F, Leotoing L, Coxam V, Guicheux J, Wittrant Y. 2009. Oxidative stress in bone remodeling and disease. Trend Mol. Med. 15: 468-477.

31. Sasaki H, Yamamoto H, Tominaga K, Masuda K, Kawai T, Teshima-Kondo S, et al. 2009. NADPH oxidase-derived reactive species are essential for differentiation of a mouse macrophage cell line (RAW264.7) into osteoclast. J. Med. Invest. 56: 33-41.

32. Srinivasan S, Koenigstein A, Joseph J, Sun L, Kalyanaraman B, Zaidi $\mathrm{M}$, et al. 2010. Role of mitochondrial reactive oxygen species in osteoclast differentiation. Ann. NY Acad. Sci. 1192: 245-252.

33. Kwon YB, Wang FF, Jang HD. 2018. Anti-osteoclastic effect of caffeic acid phenethyl ester in murine macrophages depends upon the suppression of superoxide anion production through the prevention of an active-Nox1 complex formation. $J$. Nutr. Biochem. 58: 158-168.

34. Park SJ, Jeon YJ. 2012. Dieckol from Ecklonia cava suppresses the migration and invasion of HT1080 cells by inhibiting the focal adhesion kinase pathway downstream of Rac1-ROS signaling. Mol. Cell 33: 141-149.

35. Bai XC, Lu D, Liu AL, Zhang ZM, Li XM, Zou ZP, et al.
2005. Reactive oxygen species stimulates receptor activator of NF-кB ligand expression in osteoblast. J. Biol. Chem. 280: 17497-17506.

36. Sakai E, Shimada-Sugawara M, Yamaguchi $Y$, Sakamoto $H$, Fumimoto R, Fukuma Y, et al. 2013. Fisetin inhibits osteoclastogenesis through prevention of RANKL induced ROS production by Nrf2-mediated up-regulation of phase II antioxidant enzymes. J. Pharmacol. Sci. 121: 288-298.

37. Kim KC, Kang KA, Zhang R, Piao MJ, Kim GY, Kang MY, et al. 2010. Up-regulation of Nrf2-mediated heme oxygenase-1 expression by eckol, a phlorotannin compound, through activation of Erk and PI3K/Akt. Int. J. Biochem. Cell Biol. 42: 297-305.

38. Ke K, Safder MA, Sul OJ, Kim WK, Suh JH, Joe Y, et al. 2015. Hemeoxygenase-1 maintains bone mass via attenuating a redox imbalance in osteoclast. Mol. Cell. Endocrinol. 409: 11-20.

39. Pi SH, Jeong GS, Oh HW, Kim YS, Pae HO, Chung HT, et al. 2010. Heme oxygenase-1 mediates nicotine- and lipopolysaccharide-induced expression of cyclooxygenase-2 and inducible nitric oxide synthase in human periodontal ligament cells. J. Periodontal Res. 45: 177-183. 